\title{
ON THE REDUCTION OF CERTAIN DIFFERENTIAL EQUATIONS OF
}

\section{THE SECOND ORDER*}

BY

\section{WILLIAM DUNCAN MACMILLAN}

It is proposed to investigate in this paper the reduction of the differential equations of the second order

$$
\begin{aligned}
& x_{1}^{\prime}=+p_{1} x_{1}+\sum_{j=0}^{\infty} \sum_{i=0}^{\infty} a_{i j} x_{1}^{i} x_{2}^{j}=+p_{1} x_{1}+X_{1}, \\
& x_{2}^{\prime}=-p_{2} x_{2}+\sum_{j=0}^{\infty} \sum_{i=0}^{\infty} b_{i j} x_{1}^{i} x_{2}^{j}=-p_{2} x_{2}+X_{2} \quad(i+j \geqq 2),
\end{aligned}
$$

where $p_{1}$ and $p_{2}$ are two positive numbers, and the coefficients $a_{i j}, b_{i j}$ are constants, to similar differential equations in which the right members are polynomials, by means of the linear-transcendental substitution

$$
\begin{aligned}
& x_{1}=y_{1}+\sum \sum c_{i j} y_{1}^{i} y_{2}^{j}, \\
& x_{2}=y_{2}+\sum \sum d_{i j} y_{1}^{i} y_{2}^{j} \quad(i+j \geqq 2) .
\end{aligned}
$$

Two essentially distinct cases present themselves: first, the ratio $p_{1} / p_{2}$ is irrational; second, the ratio $p_{1} / p_{2}$ is rational. It will be shown in the case $p_{1} / p_{2}=\beta$ is irrational and the number $\beta$ satisfies a rather mild condition that there exists a convergent transformation which reduces the differential equations to their linear terms. If the ratio is rational and the equations are canonical a convergent transformation exists for which the reduced differential equations have the form

$$
\begin{aligned}
& y_{1}^{\prime}=+y_{1}\left(1+Q y_{1} y_{2}\right), \\
& y_{2}^{\prime}=-y_{2}\left(1+Q y_{1} y_{2}\right),
\end{aligned}
$$

which are easily integrated. If the ratio is rational and the equations are not canonical then it is always possible to reduce the differential equations to algebraic forms but nothing can be said as to the convergence of the transformations without further knowledge of the coefficients of equations (1),

* Presented to the Society, April 21, 1916. 
except that divergent transformations are quite possible. A better statement, perhaps, would be that further knowledge of the coefficients is required before suitable algebraic forms can be determined. It seems ceitain that a knowledge of a few coefficients of terms of low degree is not sufficient.

Before entering upon the general discussion we shall show that it is no essential restriction upon the differential equations to suppose that $x_{1}$ is a factor of the right member of the first equation of (1) and that $x_{2}$ is a factor of the second equation; for if it were not so, and we take

$$
\begin{aligned}
& x_{1}=y_{1}+f_{2}\left(y_{2}\right), \\
& x_{2}=y_{2}+f_{1}\left(y_{1}\right)
\end{aligned}
$$

then $f_{1}\left(y_{1}\right)$ and $f_{2}\left(y_{2}\right)$ can be so chosen that the resulting equations in $y_{1}$ and $y_{2}$ will have this property. Making the substitution (3) in (1) and solving the resulting equations there is obtained

$$
\begin{aligned}
& \left(1-\frac{d f_{1}}{d y_{1}} \frac{d f_{2}}{d y_{2}}\right) y_{1}^{\prime}=+\left[p_{1}\left(y_{1}+f_{2}\right)+X_{1}\right]+\left[p_{2}\left(y_{2}+f_{1}\right)-X_{2}\right] \frac{d f_{2}}{d y_{2}}, \\
& \left(1-\frac{d f_{1}}{d y_{1}} \frac{d f_{2}}{d y_{2}}\right) y_{2}^{\prime}=-\left[p_{2}\left(y_{2}+f_{1}\right)-X_{2}\right]-\left[p_{1}\left(y_{1}+f_{2}\right)+X_{1}\right] \frac{d f_{1}}{d y_{1}} .
\end{aligned}
$$

The right members of these equations will carry $y_{1}$ and $y_{2}$ respectively as factors if the conditions

$$
\begin{aligned}
& {\left[p_{1} y_{1}+X_{1}\left(y_{1}, f_{1}\right)\right] \frac{d f_{1}}{d y_{1}}+\left[p_{2} f_{1}-X_{2}\left(y_{1}, f_{1}\right)\right]=0,} \\
& {\left[p_{2} y_{2}-X_{2}\left(f_{2}, y_{2}\right)\right] \frac{d f_{2}}{d y_{2}}+\left[p_{1} f_{2}+X_{1}\left(f_{2}, y_{2}\right)\right]=0}
\end{aligned}
$$

are satisfied. These conditions are obtained by putting $y_{1}=0$ in the right member of the first equation and $y_{2}=0$ in the right member of the second equation. The two equations of (5) are of the same type, the first depending only on the variable $y_{1}$ and the second only on the variable $y_{2}$. The solution of either is dominated by the solution of

$$
\left[y-\frac{M(y+f)^{2}}{1-\alpha(y+f)}\right] \frac{d f}{d y}+\beta f=\frac{M(y+f)^{2}}{1-\alpha(y+f)}
$$

where $M$ and $\alpha$ are positive constants suitably chosen, and $\beta=p_{1} / p_{2}$ or its reciprocal. If we take $f=y \phi$ it is found that the solution of $\left(5^{\prime}\right)$ is dominated by the solution of

$$
y \frac{\partial \phi}{\partial y}+(1+\beta) \phi=\frac{M y(1+\beta \phi)(1+\phi)^{2}}{1-\alpha y(1+\phi)-M y(1+\phi)^{2}},
$$


and the solution of $\left(5^{\prime \prime}\right)$ is dominated by the solution of

$$
(1+\beta) \phi=\frac{M y(1+\beta \phi)}{1-\alpha y(1+\phi)-M y(1+\phi)^{2}}
$$

which is convergent, whatever may be the value of $\beta$, since it is always positive.

If therefore $f_{1}$ and $f_{2}$ satisfy conditions (5) equations (4) are of the same form as (1) except that $y_{1}$ is a factor of the right member of the first equation and $y_{2}$ is a factor of the right member of the second. It is therefore no essential restriction to assume that the differential equations are of this form.

\section{The RAtio $p_{1} / p_{2}$ IS IRRATIONAL}

Let us suppose that $p_{1} / p_{2}$ is irrational and equal to $\gamma$. Then the equations can be written

$$
\begin{aligned}
& x_{1}^{\prime}=x_{1}\left(+1+X_{1}\right), \\
& x_{2}^{\prime}=x_{2}\left(-\gamma+X_{2}\right),
\end{aligned}
$$

where $X_{1}$ and $X_{2}$ are power series in $x_{1}$ and $x_{2}$ of order one. We make now the substitution

$$
x_{1}=y_{1}\left(1+f_{1}\right)=y_{1} e^{g_{1}}, \quad x_{2}=y_{2}\left(1+f_{2}\right)=y_{2} e^{g_{2}},
$$

where $f_{1}, f_{2}, g_{1}, g_{2}$ are power series in $y_{1}$ and $y_{2}$ vanishing for $y_{1}=y_{2}=0$. If we assume that

we obtain

$$
y_{1}^{\prime}=y_{1}, \quad y_{2}^{\prime}=-\gamma y_{2},
$$

If we set

$$
y_{1} \frac{\partial g_{1}}{\partial y_{1}}-\gamma y_{2} \frac{\partial g_{1}}{\partial y_{2}}=X_{1}\left(y_{1} e^{g_{1}}, y_{2} e^{g_{2}}\right)
$$

$$
y_{1} \frac{\partial g_{2}}{\partial y_{2}}-\gamma y_{2} \frac{\partial g_{2}}{\partial y_{2}}=X_{2}\left(y_{1} e^{g_{1}}, y_{2} e^{g_{2}}\right)
$$

$$
\begin{aligned}
& g_{1}=\sum \sum g_{i_{j}}^{(1)} y_{1}^{i} y_{2}^{j}, \\
& g_{2}=\sum \sum g_{i j}^{(2)} y_{1}^{i} y_{2}^{j},
\end{aligned}
$$

we find for the determination of the coefficients $g_{i j}^{(1)}$ and $g_{i j}^{(2)}$ the relations

$$
\begin{aligned}
& (i-j \gamma) g_{i j}^{(1)}=G_{i j}^{(1)}, \\
& (i-j \gamma) g_{i j}^{(2)}=G_{i j}^{(2)},
\end{aligned}
$$

where at each step $G_{i j}^{(1)}$ and $G_{i j}^{(2)}$ are known quantities. Since $\gamma$ is irrational none of the coefficients $(i-j \gamma)$ vanishes and there is no difficulty in determining the coefficients $g_{i j}^{(1)}$ and $g_{i j}^{(2)}$ which define the transformation.

It follows from (9) and the fact that $g_{1}$ and $g_{2}$ are power series in $y_{1}$ and $y_{2}$ 
of order one that $g_{1}=g_{2}$ if $X_{1}=X_{2}$. For the question of convergence therefore it will be sufficient to consider the single equation

$$
y_{1} \frac{\partial g}{\partial y_{1}}-\gamma y_{2} \frac{\partial g}{\partial y_{2}}=\left[\frac{1}{1-y_{1}} \cdot \frac{1}{1-y_{2}}-1\right] \frac{M}{1-g},
$$

which dominates (9). Since $\gamma$ occurs with the negative sign in the left member of this equation the solution does not have all of its signs positive and one could not be sure that the solution of (11) dominates the solution of (9). Let us consider first the solution of

$$
y_{1} \frac{\partial g}{\partial y_{1}}+\gamma y_{2} \frac{\partial g}{\partial y_{2}}=\left[\frac{1}{1-y_{1}} \cdot \frac{1}{1-y_{2}}-1\right] \frac{M}{1-g}
$$

The solution of this equation has all of its terms positive and no cancellation occurs, and the coefficients so far as they depend upon the right member of (12) have maximum values. The solution of (12) is easily obtained, for if we take

we get

$$
\omega=g-\frac{1}{2} g^{2}
$$

and consequently

$$
y_{1} \frac{\partial \omega}{\partial y_{1}}+\gamma y_{2} \frac{\partial \omega}{\partial y_{2}}=M \sum \sum y_{1}^{i} y_{2}^{j}
$$

$$
\omega=M \sum \sum \frac{y_{1}^{i} y_{2}^{j}}{i+j \gamma}
$$

Since $g=1-\sqrt{1-2 \omega}$ is expansible in powers of $\omega$ with positive coefficients it is easy to see how the function $g$ is built up and all of the coefficients so far as they depend upon the right member of (12) have maximum values.

If now we change $\gamma$ into $-\gamma$ the solution of (12) becomes the solution of (11). We have

Since the series

$$
\begin{aligned}
& g=1-\sqrt{1-2 \omega}, \\
& \omega=M \sum \sum \frac{y_{1}^{i} y_{2}^{j}}{i-j \gamma} .
\end{aligned}
$$

$$
\sum \sum \frac{y_{1}^{i} y_{2}^{j}}{|i-j \gamma|}
$$

is convergent for a certain class of irrational numbers*, $\gamma$, it follows that if we take

$$
\omega^{*}=M \sum \sum \frac{y_{1}^{i} y_{2}^{j}}{|i-j \gamma|}, \quad g^{*}=1-\sqrt{1-2 \omega^{*}}
$$

* See Bulletin of the American Mathematical Society, vol. 22 (1915), p. 26-32. 
then $g^{*}$ will be a convergent series which will dominate the solutions (9) for the coefficients of $g^{*}$ will be a maximum in so far as they depend upon the right members through $M$ and a maximum in so far as they depend upon $\gamma$ being negative.

It follows therefore, since $y_{1}=\mu_{1} e^{t}, y_{2}=\mu_{2} e^{-\gamma t}$, that $x_{1}$ and $x_{2}$ are expansible in powers of $\mu_{1} e^{t}$ and $\mu_{2} e^{-\gamma t}$, and that these expansions are convergent for any preassigned value of $t$ provided $\mu_{1}$ and $\mu_{2}$ are sufficiently small.

\section{The Ratio $p_{1} / p_{2}$ is Rational}

If the ratio $p_{1} / p_{2}$ is rational it is always possible by a linear change of the independent variable, $t$, to make $p_{1}$ and $p_{2}$ integers, and we will suppose this to have been done. Then by the substitution

we will have, since

$$
x_{1}=\xi_{1}^{p_{1}}, \quad x_{2}=\xi_{2}^{p_{2}},
$$

$$
\frac{1}{p_{1}} \frac{x_{1}^{\prime}}{x_{1}}=\frac{\xi_{1}^{\prime}}{\xi_{1}}, \quad \frac{1}{p_{2}} \frac{x_{2}^{\prime}}{x_{2}}=\frac{\xi_{2}^{\prime}}{\xi_{2}},
$$

two equations in $\xi_{1}$ and $\xi_{2}$ of the same type as (1), in which $p_{1}$ and $p_{2}$ are each unity. We may therefore suppose $p_{1}=p_{2}=1$, and the equations are

$$
\begin{aligned}
& x_{1}^{\prime}=x_{1}\left(+1+X_{1}^{(1)}\right), \\
& x_{2}^{\prime}=x_{2}\left(-1+X_{2}^{(2)}\right) .
\end{aligned}
$$

For our present purposes, however, it will be preferable to use the less specialized form

where

$$
\begin{aligned}
& x_{1}^{\prime}=+x_{1}+X_{1}, \\
& x_{2}^{\prime}=-x_{2}+X_{2},
\end{aligned}
$$

$$
X_{1}=\sum \sum a_{i j} x_{1}^{i} x_{2}^{j}, \quad X_{2}=\sum \sum b_{i j} x_{1}^{i} x_{2}^{j} \quad(i+j \geqq 2),
$$

in which it is not supposed that $x_{1}$ is a factor of $X_{1}$ nor $x_{2}$ a factor of $X_{2}$.

If, in (13), we make the linear-transcendental substitution

$$
\begin{aligned}
& x_{1}=y_{1}+\sum_{j=0}^{\infty} \sum_{i=0}^{\infty} c_{i j} y_{1}^{i} y_{2}^{j}=y_{1}+f_{1}\left(y_{1}, y_{2}\right), \\
& x_{2}=y_{2}+\sum_{i=0}^{\infty} \sum_{j=0}^{\infty} d_{i j} y_{1}^{i} y_{2}^{j}=y_{2}+f_{2}\left(y_{1}, y_{2}\right)
\end{aligned}
$$

it is found at once that the differential equations cannot be reduced to their linear terms, for it is not possible, in general, to determine the constants $c_{i j}$ and $d_{i j}$ so as to do this. It is, however, always possible to reduce them to the form 


$$
\begin{aligned}
& y_{1}^{\prime}=+y_{1}+Q_{1}^{(1)} y_{1}^{2} y_{2}+Q_{1}^{(2)} y_{1}^{3} y_{2}^{2}, \\
& y_{2}^{\prime}=-y_{2}+Q_{2}^{(1)} y_{1} y_{2}^{2}+Q_{2}^{(2)} y_{1}^{2} y_{2}^{3},
\end{aligned}
$$

in which $Q_{i}^{(j)}$ are constants provided $Q_{1}^{(1)}+Q_{2}^{(1)} \neq 0$. On substituting (14) and (15) in (13) there results

$$
\begin{gathered}
\sum_{i, j}(i-j-1) c_{i j} y_{1}^{i} y_{2}^{j}=\sum a_{i j}\left(y_{1}+f_{1}\right)^{i}\left(y_{2}+f_{2}\right)^{j}-Q_{1}^{(1)} y_{1}^{2} y_{2} \\
-Q_{1}^{(2)} y_{1}^{3} y_{2}^{2}-\left[Q_{1}^{(1)} y_{1}^{2} y_{2}+Q_{1}^{(2)} y_{1}^{3} y_{2}^{2}\right] \frac{\partial f_{1}}{\partial y_{1}} \\
-\left[Q_{2}^{(1)} y_{1} y_{2}^{2}+Q_{2}^{(2)} y_{1}^{2} y_{2}^{3}\right] \frac{\partial f_{1}}{\partial y_{2}}, \\
\sum(i-j+1) d_{i j} y_{1}^{i} y_{2}^{j}=\sum b_{i j}\left(y_{1}+f_{1}\right)^{i}\left(y_{2}+f_{2}\right)^{j}-Q_{2}^{(1)} y_{1} y_{2}^{2} \\
-Q_{2}^{(2)} y_{1}^{2} y_{2}^{3}-\left[Q_{1}^{(1)} y_{1}^{2} y_{2}+Q_{1}^{(2)} y_{1}^{3} y_{2}^{2}\right] \frac{\partial f_{2}}{\partial y_{1}} \\
-\left[Q_{2}^{(1)} y_{1} y_{2}^{2}+Q_{2}^{(2)} y_{1}^{2} y_{2}^{3}\right] \frac{\partial f_{2}}{\partial y_{2}} .
\end{gathered}
$$

From these equations it is seen that the $c_{i j}$ are readily determined except for such values of $i$ and $j$ that $i-j=+1$ and that the $d_{i j}$ are readily determined except when $i-j=-1$. Thus, up to and including terms of the second degree,

$$
\begin{aligned}
& x_{1}=y_{1}+\left[a_{20} y_{1}^{2}-a_{11} y_{1} y_{2}-\frac{1}{3} a_{02} y_{2}^{2}\right], \\
& x_{2}=y_{2}+\left[\frac{1}{3} b_{20} y_{1}^{2}+b_{11} y_{1} y_{2}-b_{02} y_{2}^{2}\right] .
\end{aligned}
$$

Using these values it is found that the terms of third degree in the right members of (16) become

$$
\begin{aligned}
R_{1}^{(3)}=\left(2 a_{20}^{2}+\frac{1}{3} a_{11} b_{20}+\right. & \left.a_{30}\right) y_{1}^{3} \\
& +\left(-a_{20} a_{11}+a_{11} b_{11}+\frac{2}{3} a_{02} b_{20}+a_{21}-Q_{1}^{(1)}\right) y_{1}^{2} y_{2} \\
& +\left(-\frac{2}{3} a_{20} a_{02}-a_{11}^{2}-a_{11} b_{02}+2 a_{02} b_{11}+a_{12}\right) y_{1} y_{2}^{2} \\
& +\left(-\frac{1}{3} a_{11} a_{02}-2 a_{02} b_{02}+a_{03}\right) y_{1}^{3}, \\
R_{2}^{(3)}=\left(2 a_{20} b_{20}+\frac{1}{3} b_{20} b_{11}\right. & \left.+b_{30}\right) y_{1}^{3} \\
& +\left(-2 b_{20} a_{11}+b_{11} a_{20}+\frac{2}{3} b_{20} b_{02}+b_{21}\right) y_{1}^{2} y_{2} \\
& +\left(-\frac{2}{3} b_{20} a_{02}-a_{11} b_{11}+b_{11} b_{02}+b_{12}-Q_{2}^{(1)}\right) y_{1} y_{2}^{2} \\
& +\left(-\frac{1}{3} b_{11} a_{02}-2 b_{02}^{2}+b_{03}\right) y_{2}^{3} .
\end{aligned}
$$

Since in the left members of (16) the coefficients of $c_{21}$ and $d_{12}$ are zero these quantities cannot be determined and they therefore remain arbitrary. From the right members therefore we must have 


$$
\begin{aligned}
Q_{1}^{(1)} & =-a_{20} a_{11}+a_{11} b_{11}+\frac{2}{3} a_{02} b_{20}+a_{21}, \\
Q_{2}^{(1)} & =-\frac{2}{3} b_{20} a_{02}-a_{11} b_{11}+b_{11} b_{02}+b_{12}, \\
Q_{1}^{(1)}+Q_{2}^{(1)} & =-a_{20} a_{11}+b_{11} b_{02}+a_{21}+b_{12},
\end{aligned}
$$

and then we obtain

$$
\begin{aligned}
& c_{30}=a_{20}^{2}+\frac{1}{6} a_{11} b_{20}+\frac{1}{2} a_{30}, \\
& c_{21}=\alpha_{1}=\text { arbitrary, } \\
& c_{12}=\frac{1}{3} a_{20} a_{02}+\frac{1}{2} a_{11}^{2}+\frac{1}{2} a_{11} b_{02}-a_{02} b_{11}-\frac{1}{2} a_{12}, \\
& c_{03}=\frac{1}{12} a_{11} a_{02}+\frac{1}{2} a_{02} b_{02}-\frac{1}{4} a_{03}, \\
& d_{30}=\frac{1}{2} a_{20} b_{20}+\frac{1}{12} b_{20} b_{11}+\frac{1}{4} b_{30}, \\
& d_{21}=-b_{20} a_{11}+\frac{1}{2} b_{11} a_{20}+\frac{1}{2} b_{11}^{2}+\frac{1}{3} b_{20} b_{02}+\frac{1}{2} b_{21}, \\
& d_{12}=\beta_{1}=\text { arbitrary, } \\
& d_{03}=\frac{1}{6} b_{11} a_{02}+b_{02}^{2}-\frac{1}{2} b_{03} .
\end{aligned}
$$

One can continue the computation as far as is desired except for the terms $y_{1}^{k+1} y_{2}^{k}$ in the first equation and $y_{1}^{k} y_{2}^{k+1}$ in the second. The coefficients $c_{k+1, k}$ $d_{k, k+1}$ (which for brevity we shall denote by $\alpha_{k}$ and $\beta_{k}$ ) cannot be determined as are the remaining coefficients. These coefficients which are left arbitrary in the terms of degree $2 k+1$ enter also in terms of higher degree and are determined by the condition that in the right members of (16) the coefficients of the terms $y_{1}^{k+2} y_{2}^{k+1}$ in the first equation and $y_{1}^{k+1} y_{2}^{k+2}$ in the second equation must vanish. Thus it is found from the coefficients of $y_{1}^{3} y_{2}^{2}$ and $y_{1}^{2} y_{2}^{3}$ that

$$
\begin{aligned}
& Q_{1}^{(2)}=-Q_{2}^{(1)} \alpha_{1}+Q_{1}^{(1)} \beta_{1}+\text { known terms, } \\
& Q_{2}^{(2)}=+Q_{2}^{(1)} \alpha_{1}-Q_{1}^{(1)} \beta_{1}+\text { known terms. }
\end{aligned}
$$

Since the determinant of the coefficients of $\alpha_{1}$ and $\beta_{1}$ in these two expressions is zero, $\alpha_{1}$ and $\beta_{1}$ cannot in general be chosen so as to make $Q_{1}^{(2)}=0, Q_{2}^{(2)}=0$. It is necessary therefore to retain these constants in the differential equations. But $k=1$ happens to be exceptional. For a general value of $k$ these two conditions are

$$
\begin{aligned}
&-\left[(k-1) Q_{1}^{(1)}+k Q_{2}^{(1)}\right] \alpha_{k}+ Q_{1}^{(1)} \beta_{k}=\text { known terms, } \\
& Q_{2}^{(1)} \alpha_{k}-\left[k Q_{1}^{(1)}+(k-1) Q_{2}^{(1)}\right] \beta_{k}=\text { known terms. }
\end{aligned}
$$

The value of the determinant $D^{(k)}$ of the coefficients of $\alpha_{k}$ and $\beta_{k}$ is

$$
D^{(k)}=k(k-1)\left(Q_{1}^{(1)}+Q_{2}^{(1)}\right)^{2} .
$$


If therefore $Q_{1}^{(1)}+Q_{2}^{(1)} \neq 0$ the determinant vanishes only if $k=0$ or $k=1$, and there is no further difficulty in the determination of the coefficients. The determination is unique aside from the fact that $\alpha_{1}$ and $\beta_{1}$ remain arbitrary. Since $\alpha_{1}$ and $\beta_{1}$ enter $Q_{1}^{(2)}$ and $Q_{2}^{(2)}$ linearly, as is seen from (18), it will be convenient to impose the relations

$$
Q_{1}^{(2)}=m Q_{1}^{(1)}, \quad Q_{2}^{(2)}=m Q_{2}^{(1)},
$$

which is always possible if $Q_{1}^{(1)}+Q_{2}^{(1)} \neq 0$, for on adding the two equations of (18) it is found that $m$ equals the sum of the known terms divided by $Q_{1}^{(1)}+Q_{2}^{(1)}$. The quantity $m$ is independent of $\alpha_{1}$ and $\beta_{1}$ and depends only upon the coefficients of the original differential equations.

With these determinations therefore the differential equations take the form

$$
\begin{aligned}
& y_{1}^{\prime}=y_{1}\left[+1+Q_{1}^{(1)}\left(1+m y_{1} y_{2}\right) y_{1} y_{2}\right], \\
& y_{2}^{\prime}=y_{2}\left[-1+Q_{2}^{(1)}\left(1+m y_{1} y_{2}\right) y_{1} y_{2}\right] .
\end{aligned}
$$

which can be integrated. On taking

$$
y_{1}=z_{1} e^{t}, \quad y_{2}=z_{2} e^{-t},
$$

the differential equations become

$$
\begin{aligned}
& z_{1}^{\prime}=Q_{1}^{(1)} z_{1}\left[z_{1} z_{2}+m z_{1}^{2} z_{2}^{2}\right], \\
& z_{2}^{\prime}=Q_{2}^{(1)} z_{2}\left[z_{1} z_{2}+m z_{1}^{2} z_{2}^{2}\right] .
\end{aligned}
$$

From these equations we get

whence

$$
\left(z_{1} z_{2}\right)^{\prime}=\left(Q_{1}^{(1)}+Q_{2}^{(1)}\right) z_{1} z_{2}\left[z_{1} z_{2}+m z_{1}^{2} z_{2}^{2}\right],
$$

so that

$$
\frac{1}{Q_{1}^{(1)}} \frac{z_{1}^{\prime}}{z_{1}}=\frac{1}{Q_{2}^{(1)}} \frac{z_{2}^{\prime}}{z_{2}}=\frac{1}{\left(Q_{1}^{(1)}+Q_{2}^{(1)}\right)} \frac{\left(z_{1} z_{2}\right)^{\prime}}{\left(z_{1} z_{2}\right)},
$$

$$
\begin{gathered}
z_{1}=c\left(z_{1} z_{2}\right)^{Q_{1}^{(1)} /\left(Q_{1}^{(1)}+Q_{2}^{(1)}\right)}, \quad z_{2}=\frac{1}{c}\left(z_{1} z_{2}\right)^{Q_{2}^{(1)} /\left(Q_{1}^{(1)}+Q_{2}^{(1)}\right)}, \\
z_{1}^{Q_{2}^{(1)}}=c^{2} z_{2}^{Q_{1}^{(1)}} .
\end{gathered}
$$

Using the function $L^{\prime}(\tau)$ which was introduced in the discussion of the differential equation of the first order* it is seen from (21) that, if $m \neq 0$,

where

$$
z_{1} z_{2}=\frac{L^{\prime}(\tau)-1}{m}
$$

$$
\tau=1-\frac{Q_{1}^{(1)}+Q_{2}^{(1)}}{m}\left(t-t_{0}\right) .
$$

\footnotetext{
* See Annals of Mathematics, vol. 19 (1917), p. 21-29.
} 
But if $m=0$ we have

$$
z_{1} z_{2}=\frac{1}{\left(Q_{1}^{(1)}+Q_{2}^{(1)}\right)\left(t_{0}-t\right)}
$$

Consequently, if $m \neq 0$, the expressions for $y_{1}$ and $y_{2}$ are

$$
\begin{aligned}
& y_{1}=c e^{t}\left(\frac{L^{\prime}(\tau)-1}{m}\right)^{Q_{1}^{(1)} /\left(Q_{1}^{(1)}+Q_{2}^{(1)}\right)}, \\
& y_{2}=\frac{1}{c} e^{-t}\left(\frac{L^{\prime}(\tau)-1}{m}\right)^{Q_{2}^{(1)} /\left(Q_{1}^{(1)}+Q_{2}^{(1)}\right)} .
\end{aligned}
$$

But if $m=0$ then

$$
\begin{aligned}
& y_{1}=\frac{c e^{t}}{\left[\left(Q_{1}^{(1)}+Q_{2}^{(1)}\right)\left(t_{0}-t\right)\right]^{Q_{1}^{(1)} /\left(Q_{1}^{(1)}+Q_{2}^{(1)}\right)},} \\
& y_{2}=\frac{\frac{1}{c} e^{-t}}{\left[\left(Q_{1}^{(1)}+Q_{2}^{(1)}\right)\left(t_{0}-t\right)\right]^{Q_{2}^{(1)} /\left(Q_{1}^{(1)}+Q_{2}^{(1)}\right)}} .
\end{aligned}
$$

It is to be observed also that equations (19) admit the integral

$$
H=y_{1}^{-\left(Q_{2}^{(1)}+m\right)} y_{2}^{\left(Q_{1}^{(1)}-m\right)}\left(1+m y_{1} y_{2}\right)^{m} e^{-1 / y_{1} y_{2}},
$$

or, in logarithmic form,

$$
\begin{aligned}
H_{1}=\left(m+Q_{2}^{(1)}\right) \log y_{1}+\left(m-Q_{1}^{(1)}\right) & \log y_{2} \\
& -m \log \left(1+m y_{1} y_{2}\right)+\frac{1}{y_{1} y_{2}} .
\end{aligned}
$$

The following example is illustrative of this transformation

$$
x_{1}^{\prime}=x_{1}+x_{1}^{2}+x_{1} x_{2}, \quad x_{2}^{\prime}=-x_{2} .
$$

The transformation defined by the relations

$$
x_{1}=\frac{y_{1} e^{-y_{2}}}{1+y_{1} y_{2} \int \frac{e^{-y_{2}}+y_{2}}{y_{2}^{2}} d y_{2}}, \quad x_{2}=y_{2},
$$

transforms the differential equations into

$$
y_{1}^{\prime}=y_{1}\left(1-y_{1} y_{2}\right), \quad y_{2}^{\prime}=-y_{2},
$$

which have the form of (19) in which $Q_{1}^{(1)}=-1, m=Q_{2}^{(1)}=0$, and it will be observed that the transformation considered as a power series in $y_{1}$ and $y_{2}$ is convergent.

It is not necessary however that the reduced equations shall have the form of (19): Indeed, it is evident from the nature of the process that any desired 
terms, of degree not less than two, could be added to the right members of (19) and the process of determining the transformation would not fail. Thus it is possible to have an infinite variety of transformations and reduced differential equations, which shows clearly that all of these transformations cannot be convergent. Consider for example, the equations

$$
\begin{aligned}
& x_{1}^{\prime}=x_{1}\left[+1+\left(1+\alpha i_{1}\right) x_{1} x_{2}+\mu x_{1}^{i_{2}+1} x^{i_{1}+1}\right], \\
& x_{2}^{\prime}=x_{2}\left[-1-\left(1+\alpha i_{2}\right) x_{1} x_{2}-\mu x_{1}^{i_{2}+1} x^{i_{1}+1}\right],
\end{aligned}
$$

in which $\alpha$ and $\mu$ are any constants and $i_{1}, i_{2}$ are positive integers or zero, but not equal. Here $Q_{1}^{(1)}+Q_{2}^{(1)}=\alpha\left(i_{1}-i_{2}\right)$ which is not zero if $\alpha$ is not zero. Consequently one can determine a transformation which will reduce (28) to (19). Equations (28) however admit the integral

$$
I=x_{1}^{-i_{2}} x_{2}^{-i_{1}} s e^{a^{a / a}}+\mu \int e^{s^{a / a}} d s,
$$

where $s^{a}=1 / x_{1} x_{2}$. If now $\mu \neq 0$ the singularities of $I$ in the neighborhood of the origin are not the same as the singularities of $(24)$. Since $I\left(y_{1} y_{2}\right)$ is not a function of $H\left(y_{1} y_{2}\right)$, their jacobian being distinct from zero, it is clear that $I$ cannot be transformed into $H$ by a convergent linear-transcendental substitution, which cannot alter the character of the singularities at the origin.* We conclude therefore that the transformation in this case is divergent if $\mu$ is distinct from zero. Furthermore, since $i_{1}$ and $i_{2}$ may be as large as we please we must conclude that the singularities of the integral in the neighborhood of the origin do not depend merely upon the coefficients of the terms of low degree in the differential equations. It would seem to be a reasonable conjecture that, if the differential equations admit an integral which is known, then there exists a convergent linear-transcendental transformation which reduces the differential equations to an algebraic type such that the reduced equations admit an integral having the same singularities at the origin as the original integral, but this is not always true, as is shown by the following example. The equations

admit the integral

$$
\begin{aligned}
& y_{1}^{\prime}=\frac{y_{1}}{1+y_{1}}\left(+1+y_{1}-y_{1} y_{2}\right), \\
& y_{2}^{\prime}=\frac{y_{2}}{1+y_{1}}\left(-1-y_{1}-y_{1} y_{2}\right)
\end{aligned}
$$

$$
H_{1}=y_{2} s e^{s / 2}+\int e^{s / 2} d s, \quad \text { where } \quad s^{2}=\frac{1}{y_{1} y_{2}} .
$$

* Dulac has considered an example in which he has shown from the law of the coefficients that the transformation in that case is divergent. See Bulletin des sciences mathématiques, vol. 37 (1913). 
Likewise the equations

admit the integral

$$
\begin{aligned}
& x_{1}^{\prime}=x_{1}\left(+1+x_{1}-x_{1} x_{2}\right), \\
& x_{2}^{\prime}=x_{2}\left(-1-x_{1}-x_{1} x_{2}\right)
\end{aligned}
$$

$$
H_{2}=x_{2} \sigma e^{\sigma^{\sigma / 2}}+\int e^{\sigma^{2} / 2} d \sigma, \quad \text { where } \quad \sigma^{2}=\frac{1}{x_{1} x_{2}} .
$$

But $(A)$ cannot be transformed into $(B)$ by a linear-transcendental substitution, for we have

$$
\frac{y_{1}^{\prime}}{y_{1}}-\frac{y_{2}^{\prime}}{y_{2}}=2, \quad \frac{\left(x_{1} x_{2}\right)^{\prime}}{\left(x_{1} x_{2}\right)^{2}}=-2 \quad \text { so that } \quad \frac{y_{1}^{\prime}}{y_{1}}-\frac{y_{2}^{\prime}}{y_{2}}=-\frac{\left(x_{1} x_{2}\right)^{\prime}}{\left(x_{1} x_{2}\right)^{2}} .
$$

That is $y_{1} / y_{2}=C e^{1 / x_{1} x_{2}}$. Obviously $y_{1} / y_{2}$ cannot be transformed into $C e^{1 / x_{1} x_{2}}$ by a linear transcendental substitution.

$$
\text { EXISTENCE OF AN INTEGRAL OF THE FORM } H=\frac{P_{1}\left(x_{1}, x_{2}\right)}{P_{2}\left(x_{1}, x_{2}\right)}
$$

It will be shown in the present section, that if there exists an integral of the form

$$
H=\frac{P_{1}\left(x_{1}, x_{2}\right)}{P_{2}\left(x_{1}, x_{2}\right)},
$$

where $P_{1}$ and $P_{2}$ are ordinary power series (and this hypothesis includes the case where the differential equations are canonical) then the transformation is convergent and the reduced equations are

$$
\begin{aligned}
& y_{1}^{\prime}=y_{1}\left(+1+Q y_{1} y_{2}\right), \\
& y_{2}^{\prime}=y_{2}\left(-1-Q y_{1} y_{2}\right) .
\end{aligned}
$$

We have already observed that it is always possible to reduce the differential equations to the form

$$
\begin{aligned}
& y_{1}^{\prime}=y_{1}\left(+1+Q_{1}^{(1)} y_{1} y_{2}+Q_{1}^{(1)} y_{1}^{2} y_{2}^{2}+Q_{1}^{(3)} y_{1}^{3} y_{2}^{3}+\cdots\right), \\
& y_{2}^{\prime}=y_{2}\left(-1+Q_{2}^{(1)} y_{1} y_{\mathrm{L}}+Q_{2}^{(2)} y_{1}^{2} y_{2}^{2}+Q_{2}^{(3)} y_{1}^{3} y_{2}^{3}+\cdots\right),
\end{aligned}
$$

whether $Q_{1}^{(1)}+Q_{2}^{(1)}=0$ or not zero, the $Q_{i}^{(j)}$ being suitably chosen constants.

On differentiating $H=P_{1} / P_{2}$ we have

$$
P_{2}\left[\frac{\partial P_{1}}{\partial x_{1}} x_{1}^{\prime}+\frac{\partial P_{1}}{\partial x_{2}} x_{2}^{\prime}\right]-P_{1}\left[\frac{\partial P_{2}}{\partial x_{1}} x_{1}^{\prime \prime}+\frac{\partial P_{2}}{\partial x_{2}} x_{2}^{\prime}\right] \equiv 0 .
$$

Let us suppose now that $P_{1}=p_{1}^{(n)}+p_{1}^{(n+1)}+\cdots, P_{2}^{\cdot}=p_{2}^{(m)}+p_{2}^{(m+1)}+\cdots$, where $p_{j}^{(k)}$ is a polynomial homogeneous in $x_{1}$ and $x_{2}$ of degree $k$. Consider the terms of lowest degree, $m+n$, in (32). We have 


$$
p_{2}^{(m)}\left(x_{1} \frac{\partial p_{1}^{(n)}}{\partial x_{1}}-x_{2} \frac{\partial p_{1}^{(n)}}{\partial x_{2}}\right)-p_{1}^{(n)}\left(x_{1} \frac{\partial p_{2}^{(m)}}{\partial x_{1}}-x_{2} \frac{\partial p_{2}^{(m)}}{\partial x_{2}}\right) \equiv 0 .
$$

Take

$$
p_{1}^{(n)}=\sum_{j=0}^{n} a_{j} x_{1}^{j} x_{2}^{n-j}, \quad p_{2}^{(m)}=\sum_{k=0}^{m} b_{k} x_{1}^{k} x_{2}^{m-k} .
$$

Then it follows from (33) that

$$
\sum_{j=0}^{n} \sum_{k=0}^{m}[2(j-k)-(n-m)] a_{j} b_{k} x_{1}^{j+k} x^{(m+n)-(j+k)} \equiv 0 .
$$

Since not every $a_{j}=0$ and not every $b_{k}=0$ we can suppose that $a_{j_{1}} \neq 0$, $b_{k_{1}} \neq 0$. Hence we must have

$$
2\left(j_{1}-k_{1}\right)-(n-m)=0,
$$

and consequently $n-m$ is an even integer, say $m=n-2 r$. For any other value of $j$, say $j_{s} \neq j_{1}$ we have

$$
2\left(j_{s}-k_{1}\right)-(n-m) \neq 0
$$

and therefore $a_{j_{s}}=0$. Likewise if $k_{s} \neq k_{1}$ we have also

$$
2\left(j_{1}-k_{s}\right)-(n-m) \neq 0
$$

and therefore $b_{k_{s}}=0$. Hence $p_{1}^{(n)}$ and $p_{2}^{(m)}$ have only one term each, and without loss of generality the coefficients of these terms can each be taken equal to unity. Furthermore we can suppose $m \neq n$. For if $m=n$ we could take the integral

$$
H_{1}=H-1=\frac{P_{1}}{P_{2}}-1=\frac{P_{1}-P_{2}}{P_{2}},
$$

for which $m \neq n$, and consequently $r \neq 0$.

We have then

$$
p_{1}^{(n)}=x_{1}^{j_{1}} x_{2}^{n-j_{1}}, \quad p_{2}^{(m)}=x^{j_{1}} x^{\left(n-j_{1}\right)}\left(x_{1} x_{2}\right) \rightarrow .
$$

Let us now make the transformation

$$
x_{1}=y_{1}+f_{1}\left(y_{1}, y_{2}\right), \quad x_{2}=y_{2}+f_{2}\left(y_{1}, y_{2}\right),
$$

such that the transformed differential equations are (31). Then $P_{1}\left(x_{1}, x_{2}\right)$ becomes

$$
S_{1}\left(y_{1}, y_{2}\right)=y_{1}^{j_{1}} y_{2}^{n-j_{1}}+s_{1}^{(n+1)}+\varepsilon_{1}^{(n+2)}+\cdots,
$$

and $P_{2}\left(x_{1}, x_{2}\right)$ becomes

$$
S_{2}\left(y_{1}, y_{2}\right)=y_{1}^{j_{1}} y_{2}^{n-j_{1}}\left(y_{1} y_{2}\right)^{-r}+s_{2}^{(m+1)}+s_{2}^{(m+2)}+\cdots .
$$


On differentiating

we have

$$
H=\frac{S_{1}\left(y_{1} y_{2}\right)}{S_{2}\left(y_{1} y_{2}\right)}
$$

$$
S_{2}\left[\frac{\partial S_{1}}{\partial y_{1}} y_{1}^{\prime}+\frac{\partial S_{1}}{\partial y_{2}} y_{2}^{\prime}\right]-S_{1}\left[\frac{\partial S_{2}}{\partial y_{1}} y_{1}^{\prime}+\frac{\partial S_{2}}{\partial y_{2}} y_{2}^{\prime}\right] \equiv 0
$$

Consider the terms of degree $n+m+1$ in the expression of (35),

On taking

$$
s_{2}^{(m+1)}\left(y_{1} \frac{\partial s_{1}^{(n)}}{\partial y_{1}}-y_{2} \frac{\partial s_{1}^{(n)}}{\partial y_{2}}\right)-s_{1}^{(n+1)}\left(y_{1} \frac{\partial s_{2}^{(m)}}{\partial y_{1}}-y_{2} \frac{\partial s_{2}^{(m)}}{\partial y_{2}}\right) \equiv 0
$$

$$
s_{1}^{(n+1)}=\sum_{k=0}^{n+1} c_{k} y_{1}^{k} y_{2}^{n+1-k}, \quad s_{2}^{(m+1)}=\sum_{j=0}^{m+1} d_{j} y_{1}^{j} y_{2}^{m+1-j}
$$

we find, after removing a common factor,

$\sum_{k=0}^{n+1}\left[2\left(k-j_{1}\right)-1\right] c_{k} y_{1}^{k-r} y_{2}^{n+1-r-k}-\sum_{j=1}^{n+1-2 r}\left[2\left(j-j_{1}\right)+2 r-1\right] d_{j} y_{1}^{j} y^{n+1-j-2 r}$.

If $r$ is positive the first $\sum$ contains more terms than the second, and if $r$ is negative the second contains more than the first. Let us suppose $r>0$ (the result is the same for $r<0$ ). Then $c_{k}=0$ for $k=0, \cdots, r-1$, $n+2-r, \cdots, n+1$. If now we take $k-r=j$ for the remaining terms we get

$$
\sum_{j=0}^{n+1-2 r}\left[2\left(j-j_{1}+r\right)-1\right]\left[c_{j}-d_{j}\right] y_{1}^{j} y_{2}^{n+1-2 r-j} \equiv 0
$$

and therefore $c_{j}=d_{j}$, since $2\left(j-j_{1}+r\right)-1$ is an odd integer. Hence

$$
s_{1}^{(n+1)}=\left(y_{1} y_{2}\right)^{r} s_{2}^{(m+1)} \text {. }
$$

From the terms of degree $m+n+2$ we obtain

$$
\begin{aligned}
& s_{2}^{(m)}\left(y_{1} \frac{\partial s_{1}^{(n+2)}}{\partial y_{1}}-y_{2} \frac{\partial s_{1}^{(n+2)}}{\partial y_{2}}\right)-s_{1}^{(n)}\left(y_{1} \frac{\partial s_{2}^{(m+2)}}{\partial y_{2}}-y_{2} \frac{\partial s_{2}^{(m+2)}}{\partial y_{2}}\right) \\
& +s_{2}^{(m+1)}\left(y_{1} \frac{\partial s^{(n+1)}}{\partial y_{1}}-y_{2} \frac{\partial s^{(n+1)}}{\partial y_{2}}\right)-s_{1}^{(n+1)}\left(y_{1} \frac{\partial s_{2}^{(m+1)}}{\partial y_{1}}-y_{2} \frac{\partial s_{2}^{(m+1)}}{\partial y_{2}}\right) \\
& +s_{2}^{(m+2)}\left(y_{1} \frac{\partial s_{1}^{(n)}}{\partial y_{1}}-y_{2} \frac{\partial s_{1}^{(n)}}{\partial y_{2}}\right)-s_{1}^{(n+2)}\left(y_{1} \frac{\partial s_{2}^{(m)}}{\partial y_{1}}-y_{2} \frac{\partial s_{2}^{(m)}}{\partial y_{2}}\right) \\
& +r\left(Q_{1}^{(1)}+Q_{2}^{(1)}\right) y_{1}^{2 j_{1}-r+1} y_{2}^{\left(n-j_{1}\right)-r+1} \equiv 0 .
\end{aligned}
$$

The terms of the second line vanish by themselves. If we take 


$$
s_{1}^{(n+2)}=\sum_{k=0}^{n+2} e_{k} y_{1}^{k} y_{2}^{n+2-k}, \quad s_{2}^{(m+2)}=\sum_{j=2}^{m+1} f_{j} y_{1}^{j} y_{2}^{m+2-j}
$$

the identity (37) is easily reduced to

$\sum_{j=0}^{n+2-2 r} 2\left(j-j_{1}+r-1\right)\left(e_{j}-f_{j}\right) y_{1}^{j} y_{2}^{n+2-2 r-j} \equiv-r\left(Q_{1}^{(1)}+Q_{2}^{(2)}\right) y^{j_{1}-r+1} y_{2}^{n-j_{1}-r+1}$.

For $j=j_{1}-r+1$ the coefficient of the left member vanishes, and the same must therefore be true of the right member. Therefore $Q_{1}^{(1)}+Q_{2}^{(2)}=0$, since $r \neq 0$. For the other values of $j$ we must have $e_{j}=f_{j}$. Hence

$$
s_{1}^{(n+2)}=\left(y_{1} y_{2}\right)^{r} s_{2}^{(m+2)}+a y_{1}^{j_{1}+1} y_{2}^{n-j_{1}+1},
$$

where $a$ is some constant.

Proceeding in this manner it can be shown by induction that

$$
Q_{1}^{(i)}+Q_{2}^{(i)}=0 \quad i=1, \cdots, \infty, \quad \text { and } \quad S_{1}=\left(y_{1} y_{2}\right)^{r} A\left(y_{1} y_{2}\right) S_{2},
$$

where $A$ is an ordinary power series in the product $\left(y_{1} y_{2}\right)$, and this result is readily verified, for if we take

$$
\begin{gathered}
y_{1}^{\prime}=y_{1}\left(+1+Q^{(1)} y_{1} y_{2}+Q^{(2)} y_{1}^{2} y_{2}^{2}+\cdots\right), \\
y_{2}^{\prime}=y_{2}\left(-1-Q^{(1)} y_{1} y_{2}-Q^{(2)} y_{1}^{2} y_{2}^{2}-\cdots\right), \\
S_{1}=A S_{2},
\end{gathered}
$$

where $A$ is an arbitrary power series in $\left(y_{1} y_{2}\right)$, and substitute in (35) it is found at once that this equation is satisfied.

It follows therefore if there exists an integral of the original differential equations of the form

$$
H=\frac{P_{1}\left(x_{1} x_{2}\right)}{P_{2}\left(x_{1} x_{2}\right)},
$$

where $P_{1}$ and $P_{2}$ are power series in $x_{1}$ and $x_{2}$, that a linear transcendental substitution which transforms the differential equations into the form (31) is such as to make $Q_{1}^{(i)}+Q_{2}^{(i)}=0, i=1, \cdots, \infty$, and furthermore transtorms the quotient $P_{1} / P_{2}$ into a power series in the product $\left(y_{1} y_{2}\right)$.

Returning now to equation (18) and putting

$$
Q_{1}^{(1)}=-Q_{2}^{(1)}=Q, \quad Q_{1}^{(2)}=-Q_{2}^{(2)}=0,
$$

it is clear that if $Q \neq 0$ the sum $\left(\alpha_{1}+\beta_{1}\right)$ can be chosen so as to satisfy both equations. The difference $\left(\alpha_{1}-\beta_{1}\right)$ remains arbitrary. From (18') we have in general 


$$
\begin{array}{cc}
Q_{1}^{(k+1)}=0=\left[(k-1) Q_{1}^{(1)}+k Q_{2}^{(1)}\right] \alpha_{k} & Q_{1}^{(1)} \beta_{k} \\
Q_{2}^{(k+1)}=0= & + \text { known terms, } \\
& -Q_{2}^{(1)} \alpha_{k}+\left[k\left(Q_{1}^{1)}+(k-1) Q_{2}^{(1)}\right] \beta_{k}\right. \\
+ \text { known terms. }
\end{array}
$$

Since $Q_{1}^{(k+1)}+Q_{2}^{(k+1)}=0$ the second equation is merely the negative of the first. Either of these equations gives

$$
Q\left(\alpha_{k}+\beta_{k}\right)=\text { known terms. }
$$

Thus $\alpha_{k}+\beta_{k}$ is determined by the choice $Q_{1}^{(k+1)}=Q_{2}^{(k+1)}=0$. The difference $\alpha_{k}-\beta_{k}$ is undetermined. There are therefore infinitely many undetermined constants in the transformation, but whatever may be the value of these constants the reduced differential equations are

$$
\begin{aligned}
& y_{1}^{\prime}=+y_{1}\left(1+Q y_{1} y_{2}\right), \\
& y_{2}^{\prime}=-y_{2}\left(1+Q y_{1} y_{2}\right) .
\end{aligned}
$$

From these equations are derived the integral, $y_{1} y_{2}=c$, and therefore

$$
\begin{aligned}
& y_{1}=\sqrt{c} e^{(1+Q c)\left(t-t_{0}\right)}, \\
& y_{2}=\sqrt{c} e^{-(1+Q c)\left(t-t_{0}\right)} .
\end{aligned}
$$

Proof of Convergence. In the substitution (2) we will denote the sum of all terms in $x_{1}$ which have the form $y_{1}\left(y_{1} y_{2}\right)^{8}$ by $y_{1} \phi_{1}$, and the sum of all terms in $x_{2}$ which have the form $y_{2}\left(y_{1} y_{2}\right)^{s}$ by $y_{2} \phi_{2}$. Then we can write

$$
\begin{aligned}
& x_{1}=y_{1}\left(1+\phi_{1}\right)+f_{1}{ }^{*}, \\
& x_{2}=y_{2}\left(1+\phi_{2}\right)+f_{2}{ }^{*} ;
\end{aligned}
$$

and from the process just described it is seen that the sum $\phi_{1}+\phi_{2}$ is determined by the condition that the reduced equations shall have the form (40), while the difference $\phi_{1}-\phi_{2}$ is undetermined.

The result of substituting (40) and (42) in the original equations (1) is

$$
\begin{aligned}
& y_{1} \frac{\partial f_{1} *}{\partial y_{1}}-y_{2} \frac{\partial f_{1} *}{\partial y_{2}}-f_{1}^{*}=\frac{X_{1}+Q y_{1}^{2} y_{2}\left(1+\phi_{1}\right)+Q y_{1} y_{2} f_{1} *}{1-Q y_{1} y_{2}}=R_{1}, \\
& y_{1} \frac{\partial f_{2} *}{\partial y_{1}}-y_{2} \frac{\partial f_{2}}{\partial y_{2}}-f_{2} *=-\frac{X_{2}+Q y_{1} y_{2}^{2}\left(1+\phi_{2}\right)+Q y_{1} y_{2} f_{2}^{*}}{1-Q y_{1} y_{2}}=R_{2},
\end{aligned}
$$

since

$$
\begin{gathered}
y_{1} \frac{\partial\left(y_{1} \phi_{1}\right)}{\partial y_{1}}-y_{2} \frac{\partial\left(y_{1} \phi_{1}\right)}{\partial y_{2}}-\left(y_{1} \phi_{1}\right) \equiv 0 \\
y_{1} \frac{\partial\left(y_{2} \phi_{2}\right)}{\partial y_{1}}-y_{2} \frac{\partial\left(y_{2} \phi_{2}\right)}{\partial y_{2}}+\left(y_{2} \phi_{2}\right) \equiv 0 .
\end{gathered}
$$


Taking now

$$
\begin{aligned}
& f_{1}{ }^{*}=\sum \sum m_{i j} y_{1}^{i} y_{2}^{j}, \quad i+j \geqq 2 \quad \text { but } \quad i \neq j+1, \\
& f_{2}{ }^{*}=\sum \sum n_{i j} y_{1}^{i} y_{2}^{j}, \quad i+j \geqq 2 \text { but } j \neq i+1 \text {, }
\end{aligned}
$$

there results

$$
\begin{aligned}
& \sum \sum(i-j-1) m_{i j} y_{1}^{i} y_{2}^{j}=R_{1}, \\
& \sum \sum(i-j+1) n_{i j} y_{1}^{i} y_{2}^{j}=R_{2},
\end{aligned}
$$

from which the coefficients of the left members can be computed successively if $Q$ is properly chosen.

If, however, we add to the first equation

and subtract

$$
\sum m_{i, i-1} y_{1}^{i} y_{2}^{i-1}=y_{1}\left(1+\phi_{1}\right) \text {, }
$$

$$
\sum n_{i, i+1} y_{1}^{i} y_{2}^{i+1}=y_{2}\left(1+\phi_{2}\right) \text {, }
$$

from the second equation we have

$$
\begin{aligned}
& \sum m_{i, i-1} y_{1}^{i} y_{2}^{i-1}+\sum^{\prime}(i-j-1) m_{i j} y_{1}^{i} y_{2}^{j}=+y_{1}\left(1+\phi_{1}\right)+R_{1}, \\
& \sum n_{i, i+1} y_{1}^{i} y_{2}^{i+1}+\sum^{\prime}(i-j+1) n_{i j} y_{1}^{i} y_{2}^{j}=-y_{2}\left(1+\phi_{2}\right)-R_{2} .
\end{aligned}
$$

The coefficients of the left members of these equations (45) also can be determined so that the equations are satisfied identically whatever $\phi_{1}$ and $\phi_{2}$ in the right members may be, and the solutions thus obtained are convergent; for the solutions thus obtained are dominated by the solutions of the equations which are obtained by replacing each $(i-j+1)$ in the left members of the first equation by +1 and each $(i-j+1)$ of the second equation by -1 and taking all the coefficients in the expansions of $R_{1}$ and $R_{2}$ with the positive sign, and the solutions of the equations thus modified are known to be convergent.

The coefficients $m_{i j}$ and $n_{i j}$ thus obtained from (45) will be functions of $\phi_{1}$ and $\phi_{2}$. But we must have

$$
\begin{aligned}
& \sum m_{i, i-1} y_{1}^{i} y_{2}^{i-1}=y_{1}\left(1+\phi_{1}\right), \\
& \sum n_{i, i+1} y_{1}^{i} y_{2}^{i+1}=y_{2}\left(1+\phi_{2}\right),
\end{aligned}
$$

the left members of which are convergent series. It will be observed that in (45) the factor $\left(1+\phi_{1}\right)$ is always associated with $y_{1}$ except in the product $\left(y_{1} y_{2}\right)$ and the factor $\left(1+\phi_{2}\right)$ is associated in the same way with $y_{2}$. Hence the first equation of (46) contains $y_{1}\left(1+\phi_{1}\right)$ as a factor and the second contains $y_{2}\left(1+\phi_{2}\right)$ as a factor. Removing these factors and giving the $m_{i j}$ and $n_{i j}$ the values obtained from the solution of (45) we get

$$
\begin{aligned}
& 0=\left[Q\left(1+\phi_{1}\right)\left(1+\phi_{2}\right)-Q\right] y_{1} y_{2}+[] y_{1}^{2} y_{2}^{2}+\cdots, \\
& 0=\left[Q\left(1+\phi_{1}\right)\left(1+\phi_{2}\right)-Q\right] y_{1} y_{2}+[] y_{1}^{2} y_{2}^{2}+\cdots .
\end{aligned}
$$


On removing the factor $y_{1} y_{2}$ we have

$$
\begin{aligned}
& 0=Q\left(\phi_{1}+\phi_{2}\right)+[] y_{1} y_{2}+\cdots, \\
& 0=Q\left(\phi_{1}+\phi_{2}\right)+[] y_{1} y_{2}+\cdots .
\end{aligned}
$$

These equations impose a condition only on $\phi_{1}+\phi_{2}$ and they must therefore be identical. We have already seen that we can take $\phi_{1}-\phi_{2}$ arbitrary. Hence we have

$$
\begin{aligned}
& 0=Q\left(\phi_{1}+\phi_{2}\right)+[] y_{1} y_{2}+\cdots, \\
& 0=\phi_{1}-\phi_{2}+y_{1} y_{2} \psi\left(y_{1} y_{2}\right)+\cdots .
\end{aligned}
$$

If the arbitrary function $\psi$ is given and is a convergent ordinary power series equations (48) admit a unique convergent solution for $\phi_{1}$ and $\phi_{2}$.

It might seem since $m_{i j}$ and $n_{i j}$ are functions of $\phi_{1}$ and $\phi_{2}$ that when the values thus derived for $\phi_{1}$ and $\phi_{2}$ are substituted in $f_{1}{ }^{*}$ and $f_{2}{ }^{*}$ they would then contain terms of the type $y_{1}^{i} y_{2}^{i-1}, y_{1}^{i} y_{2}^{i+1}$ respectively, but this is clearly not so, since the difference between the exponents of $y_{1}$ and $y_{2}$ in any term is not altered by multiplying the term by any power of $\left(y_{1} y_{2}\right)$.

Example.-As an illustration of this method of solution let us consider the equation $d^{2} \phi / d t^{2}+\sin \phi=0$, whence $d^{2} \phi / d t^{2}=-\phi+\frac{1}{6} \phi^{3}-{ }_{1}^{\frac{1}{2}} \phi^{5}+\cdots$. This equation will be reduced to the normal form if we take

$$
\phi=\frac{1}{2}\left(\xi_{1}+i \xi_{2}\right), \quad d \phi / d t=\frac{1}{2}\left(i \xi_{1}+\xi_{2}\right),
$$

where $i=\sqrt{-1}$. The equations then are, if we take $i t=\tau$,

where

$$
\xi_{1}^{\prime}=\xi_{1}-f, \quad \xi_{2}^{\prime}=-\xi_{2}-i f,
$$

$$
f=\frac{1}{48}\left(\xi_{1}+i \xi_{2}\right)^{3}-\frac{1}{38} \overline{80}\left(\xi_{1}+i \xi_{2}\right)^{5}+\cdots .
$$

On making the linear-transcendental substitution

$$
\begin{aligned}
& \xi_{1}=\eta_{1}+\left[-\frac{1}{96} \eta_{1}^{3}-\frac{3 i}{128} \eta_{1}^{2} \eta_{2}-\frac{1}{32} \eta_{1} \eta_{2}^{2}-\frac{i}{192} \eta_{2}^{3}\right]+\cdots, \\
& \xi_{2}=\eta_{2}+\left[-\frac{i}{192} \eta_{1}^{3}+\frac{1}{32} \eta_{1}^{2} \eta_{2}-\frac{3 i}{128} \eta_{1} \eta_{2}^{2}+\frac{1}{96} \eta_{2}^{3}\right]+\cdots,
\end{aligned}
$$

the reduced equations are found to be

$$
\eta_{1}^{\prime}=\eta_{1}\left(1-\frac{i}{16} \eta_{1} \eta_{2}\right), \quad \eta_{2}^{\prime}=-\eta_{2}\left(1-\frac{i}{16} \eta_{1} \eta_{2}\right)
$$

The integral of these equations is $\eta_{1} \eta_{2}=c$. It is convenient to take

$$
c=-16 i \gamma \text {. }
$$


Then we find

$$
\eta_{1}=\sqrt{c} e^{(1-\gamma) \tau+a}, \quad \eta_{2}=\sqrt{c} e^{-(1-\gamma) \tau-a} .
$$

If now we take $\alpha=-\pi i / 4$ and replace $\tau$ by $i t$ we find

$\phi=\frac{1}{2}\left(\xi_{1}+i \xi_{2}\right)=[4 \sin (1-\gamma) t] \gamma^{1 / 2}$

$$
+\left[\frac{1}{2} \sin (1-\gamma) t+\frac{1}{3} \sin 3(1-\gamma) t\right] \gamma^{3 / 2}+\cdots .
$$

Since $t$ does not enter explicitly in the original differential equation one can replace $t$ by $\left(t-t_{0}\right)$ in the above solution in which $\phi=0$ for $t=0$. 